\title{
Five fake ultra-luminous X-ray sources
}

\author{
C. M. Gutiérrez
}

\author{
Instituto de Astrofísica de Canarias, Tenerife, Spain \\ e-mail: cgc@iac.es
}

Received 24 September 2012 / Accepted 8 November 2012

\begin{abstract}
Context. The nature of ultra-luminous X-ray (ULX) sources is still a matter of debate. Studies of their counterparts in other spectral ranges will help to clarify the nature of those sources.

Aims. Our goal was to identify and characterize the optical counterparts of five sources catalogued as ULXs.

Methods. We obtained optical imaging and intermediate resolution spectroscopy of these optical counterparts.

Results. We demonstrate that five sources previously catalogued as ULXs, two are foreground stars in our galaxy and three are background active galactic nuclei.
\end{abstract}

Key words. quasars: general - X-rays: general - stars: general

\section{Introduction}

The nature of ultra-luminous X-ray (ULX) sources is still unclear. These sources appear as point-like objects distributed around nearby (parent) galaxies and have X-ray luminosities (assuming the redshift of the parent galaxy and isotropic emission) above the Eddington limit for a stellar mass black hole (BH). Although there is a wide consensus that they are the manifestation of compact sources that accrete matter, their luminosities are higher than those expected from normal X-ray binary systems. One exciting scenario invokes the existence of a new class of X-ray binaries with $\mathrm{BH}$ with masses $20-10000 M_{\odot}$. Other explanations invoke a stellar mass $\mathrm{BH}$ with nonstandard accretion at super-Eddington rates or with beamed emission (King et al. 2001; Kording et al. 2002).

The identification and characterization of optical counterparts of ULXs offer a good approach to clarify the nature of these sources. Many ULXs do not show a clear optical counterpart or reside in star-forming or crowded regions, which complicates the correct identification of their counterparts. The spatial resolution of images obtained with the modern satellites Chandra and XMM-Newton, similar to those of optical images with ground-based telescopes, alleviates that problem. So far, very few of these optical counterparts have been identified and characterized spectroscopically. In these cases, the emission can be explained as contribution of the companion star (usually $\mathrm{O}$ or B types) and irradiation from an accretion disk onto a massive $\left(100-1000 M_{\odot}\right)$ BH (NGC 5408 X-1 Grise et al. 2012; Holmberg II X-1 Tao et al. 2012; NGC 1313 X-1 Yang et al. 2011). In other cases (IC 342 X-1 Cseh et al. 2012) the dominant features are those from a nebula around a $\mathrm{BH}$ with mass $\leq(1.0 \pm 0.3) \times 10^{3} M_{\odot}$. In ESO 243-49 HLX-1 the optical counterpart could be modelled as the emission of an irradiated accretion disk plus an unresolved cluster of stars (e.g. Farrel et al. 2012). These observations are still very rare, so it is required to observe more objects to build a representative sample to establish the physical properties of these objects on a firm basis.
Here, we continue our previous efforts (Arp et al. 2004; Ghosh et al. 2009; Gutiérrez 2006; Gutiérrez \& López-Corredoira 2005, 2007; López-Corredoira \& Gutiérrez 2006) to identify and characterize optical counterparts of ULXs. We put special emphasis on the identification of foreground and background contaminants to establish a correct census and statistics of real ULXs. The targets for the work presented here were selected from the catalogues of ULX candidates by Liu (2011) and Swartz et al. (2011). The main database for optical images was the SDSS survey. For cases where the field of a given ULX was not covered by that survey, we inspected $\operatorname{DSS}^{1}$ images. Some of the fields also have available images in HST archives. The strategy was to identify a likely optical counterpart compatible with the spatial uncertainties of the X-ray and optical coordinate and finding out its nature by taking long-slit spectra with intermediate resolution. The five X-ray sources previously catalogued as ULXs that are analysed in this paper lie on regions sparsely populated in the optical, and the association between X-ray source and their respective optical counterparts is very conspicous. The paper follows a standard structure presenting the sample selection and observations (Sect. 2), the analysis of each source (Sect. 3), and the discussion and conclusions (Sect. 4).

\section{Sample selection and observations}

Liu (2011) listed 479 ULXs detected by Chandra within 188 host galaxies. Of these, 74 are extreme ULXs, i.e. sources with $L_{\mathrm{X}}(0.3-8 \mathrm{keV})>10^{40} \mathrm{erg} \mathrm{s}^{-1}$ at distances within $2 \mathrm{D}_{25}$ isophotes of the host galaxies. Swartz et al. (2011) provided a Chandra- and XMM-Newton-based catalogue that presents 107 potential ULXs in galaxies at distances between 3.1 and 14.5 Mpc. The objects analysed in this work were finally selected from those with a likely optical counterpart bright enough for the instrumental configuration that were well-positioned in

1 http://archive.stsci.edu/cgi-bin/dss_form/ 
Table 1. ULX sources.

\begin{tabular}{|c|c|c|c|c|c|c|c|}
\hline Name & RA (J2000) & Dec (J2000) & $\begin{array}{l}\text { Angular c } \\
\text { (arcmin) }\end{array}$ & $\begin{array}{r}\text { stance } \\
\left(D_{25}\right)\end{array}$ & $\begin{array}{c}L_{\mathrm{X}} \\
\left(\mathrm{erg} \mathrm{s}^{-1}\right) \\
\end{array}$ & $\begin{array}{l}t_{\text {exp }} \\
(\mathrm{s})\end{array}$ & $\begin{array}{c}\text { Offset } \\
(\operatorname{arcsec})\end{array}$ \\
\hline NGC $1068-\mathrm{X} 2$ & $02: 42: 44.42$ & $-00: 06: 33.3$ & 5.8 & 1.94 & $1.18 \times 10^{40}$ & 120 & 0.34 \\
\hline NGC 2986-X1 & 09:44:14.28 & $-21: 14: 32.0$ & 2.2 & 1.57 & $2.05 \times 10^{40}$ & 400 & 0.26 \\
\hline NGC 5322-X1 & $13: 48: 59.46$ & $+60: 14: 58.0$ & 4.0 & 1.87 & $1.75 \times 10^{40}$ & 1800 & 0.19 \\
\hline NGC 2500-X2 & 08:01:57.85 & $+50: 43: 39.5$ & 0.9 & 0.69 & $1.1 \times 10^{39}$ & 1800 & 0.35 \\
\hline NGC 4150-X2 & $12: 10: 34.77$ & $+30: 23: 58.3$ & 0.3 & 0.28 & $1.08 \times 10^{40}$ & 1800 & 1.01 \\
\hline
\end{tabular}
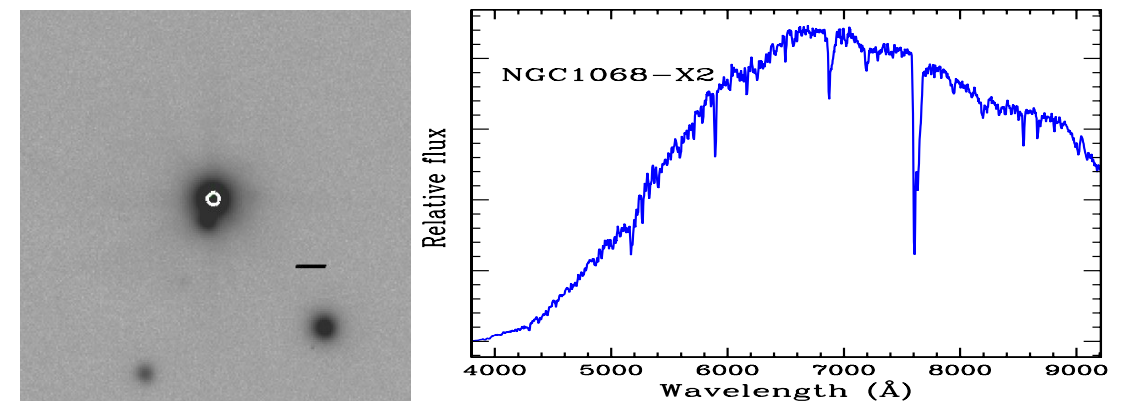

Fig. 1. (Left) Optical image centred on the position of the ULX candidate NGC 1068-X2. North is up and east to the left. The radius of the circle is centred on the X-ray nominal position and is 1 arcsec. The solid horizontal line is shown for scale and has 5 arcsec. (Right) Spectra of the optical counterpart of the ULX candidate. For visual purposes, the spectrum has been slightly smoothed. the sky for the allocated observing dates (December 2011). Considering the relatively poor observing conditions in the spectroscopic run (see below), we decided to observe those objects with a brighter optical counterpart that were situated in relatively isolated regions.

Our five sources are NGC 1068-X2, NGC 2986-X1, and NGC 5322-X1 selected from the catalogue by Liu, and NGC 2500-X2 and NGC 4150-X2 selected from Swartz et al. The first three sources are at distances between 1 and $2 \mathrm{D}_{25}$ from the centre of their respective host galaxies. For these sources Liu estimated a fraction of contaminants of 59\% (16 out of 27). The host galaxies of these candidates are within the range 10.1-32.3 Mpc, and then the distance modulus are between 30.0 and 32.5 mag. Considering the apparent magnitudes of the optical counterparts, these magnitudes would correspond to objects much more brighter than those attributed to the confirmed optical counterparts of real ULXs (Tao et al. 2011). If the optical counterpart of these ULXs were dominated by the light of the donor star, the expected optical emission would be fainter than $\sim 23$ for a main-sequence star, and $\sim 18$ mag for the most hypergiant stars known. On the other hand, Copperwheat et al. (2005) have shown how the irradiated accretion disc can have luminosities of up to $M_{V} \sim-11 \mathrm{mag}$.

\subsection{Observations}

The spectroscopic observations presented here were taken in a single night in December 2011 on the $4.2 \mathrm{~m}$ William Herschel Telescope (WHT) using the low-resolution spectrometer ACAM. The night was clear but the seeing was quite unstable and in general poor, with values for the observations presented here in the range 1.5-2.5 arcsec. The spectra were taken at relatively high elevation (airmasses in the range 1.1-1.6). We took long-slit spectra with the width of the slit fitted to match the respective values of the seeing. The instrumental configuration covers the range $3700-8600 \AA$ and provides a sampling of $3.3 \AA$ pixel $^{-1}$, although the effective spectral resolutions were in the range 12-24 $\AA$ as measured in arc and sky lines. For wavelength calibration we took $\mathrm{Cu}-\mathrm{Ar}$ and $\mathrm{Cu}-\mathrm{Ne}$ lamps. The calibration was obtained by fitting the position of $\sim 30$ spectral lines, and has a typical uncertainty of $0.3 \AA$ (for wavelengths shorter than $5500 \AA$ this uncertainty is larger due to the low number of arc lines in that range). Owing to the low emission of the continuum illumination lamp in the blue part of the spectra, we did not apply any flat field correction. This has little effect on the extracted spectra. The fields centred in the position of the sources NGC 1068-X2 and NGC 2986-X1 were observed with the IAC 80 telescope equipped with the CAMELOT camera. In the first case, the possible optical counterpart was saturated in SDSS images which prevented a reliable estimation of position and photometry. In the second case, there were no SDSS images and the optical counterpart had been identified with DSS images. Images in $g, r$, and $i$ were taken in February and March 2012. Typical uncertainties in the photometry derived from these observations are $0.05 \mathrm{mag}$. The spectra and images were reduced using IRAF ${ }^{2}$.

\section{Analysis}

Table 1 presents the basic information for the five ULX candidates. For the first three sources, those from the catalogue by Liu, we use the name given by this author. The last two have been selected from the catalogue by Swartz et al., who did not give a specific name to the sources, so we denoted them by the name of the respective host galaxy followed by a number indicating the order in which they are listed in this catalogue (i.e. NGC 2500X2 and NGC 4150-X2). The separations (Cols. 4 and 5) between the source and their host galaxy is given in arcmins and in units of $D_{25}$ from their respective putative parent galaxies. The last two columns indicate the exposure time for the spectroscopic observation and the offsets between the X-ray and optical coordinates. All offsets are below $\sim 1$ arcsec. These are probably dominated by the uncertainty in the X-ray positions. In fact, the astrometric uncertainties quoted by Liu and Swartz et al. are $\sim 1$ and 0.3 arcsec, respectively. Figures $1-5$ present the optical images and spectra of each source.

\subsection{NGC 1068-X2}

SDSS images show a very bright saturated source compatible with the Chandra coordinates of the X-ray source. To estimate a reliable astrometry and brightness of the source, we took $g$,

2 IRAF is the Image Reduction and Analysis Facility, written and supported by the IRAF programming group at the national Optical Astronomy Observatories (NOAO) in Tucson, Arizona. 

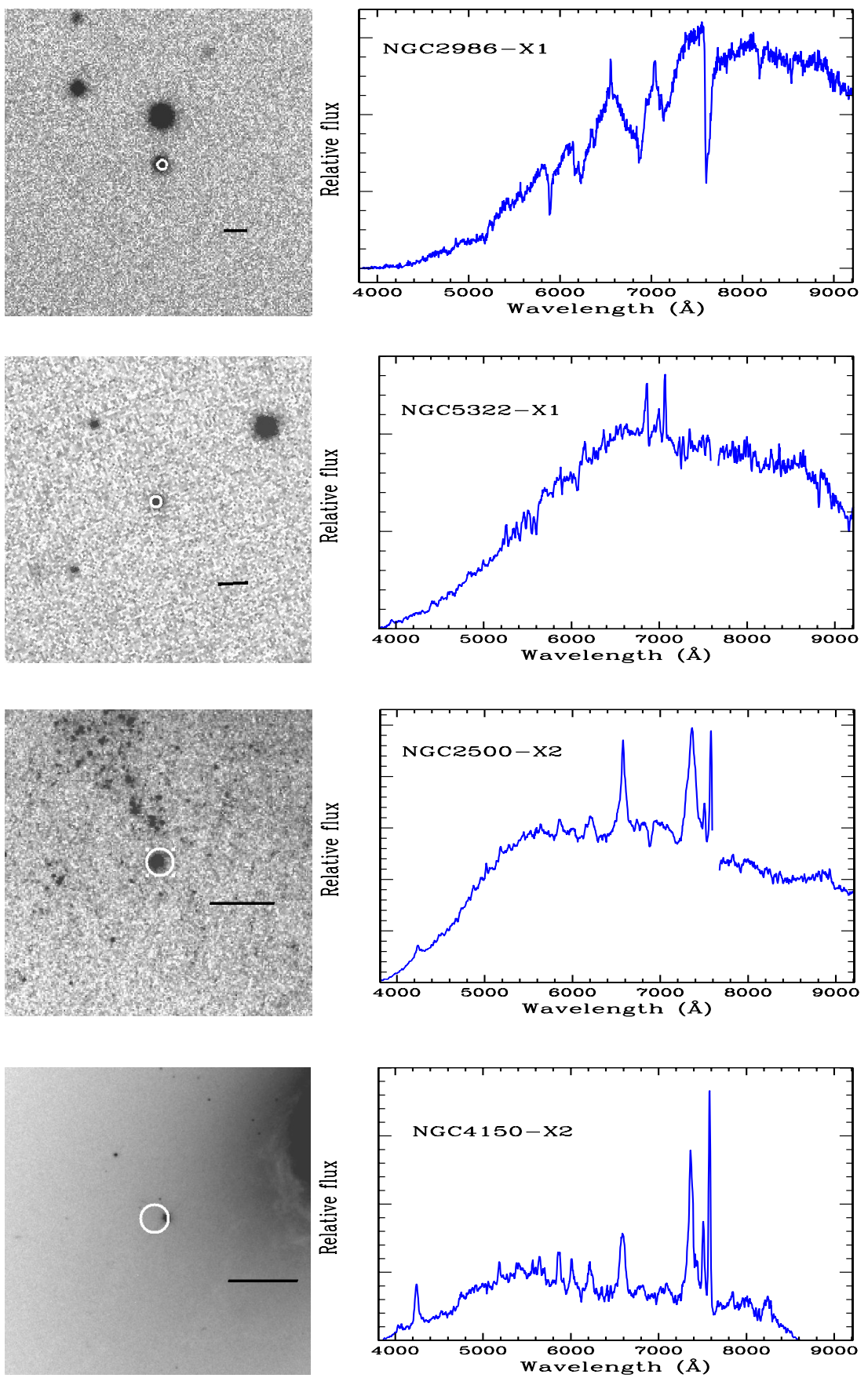

Fig. 2. Idem for the source NGC 2986-X1.

Fig. 3. Idem for the source NGC 5322-X1. In this and in the following figure a section of the spectrum centred at $\sim 7600 \AA$ has been omitted due to the poor subtraction of the telluric band.

Fig. 4. Idem for the source NGC 2500-X2.

Fig. 5. Idem for the source NGC 4150-X2. $r$, and $i$ images of the field with the IAC80 telescope. The images confirm the point-like nature of the source and allow one to determine a separation between optical and X-ray coordinates of 0.34 arcsec. The magnitudes are 12.80, 11.95, and 11.66 in the $g, r$, and $i$ filters. The spectrum corresponds to those of a $\mathrm{K} 0$ star. On the basis of the spectral type and these magnitudes, we estimate a distance to the star of $191 \mathrm{pc}$. The uncertainty on this distance is $\sim 20 \%$ and comes mainly from the uncertainty in the estimation of the spectral type. We conclude that this ULX candidate is in fact an X-ray source with a luminosity of $2.4_{-0.9}^{+1.1} \times 10^{30} \mathrm{erg} \mathrm{s}^{-1}$ in our own galaxy. The X-ray-to-optical flux is $\sim-2.0$, which is within the range measured in a set of K stars by Stocke et al. (1991). The source named NGC 1068 X-4 in the ROSAT-based catalogue by Liu \& Bregman (2005) is at 2.7 arcsec from the Chandra coordinates. This separation is compatible with ROSAT uncertainty in positions, while the respective luminosities agree within a factor 2 , so we consider both to be the same source.

Another point-like object about 3 magnitudes fainter is situated at a distance of 3.5 arcsec to the south of the optical counterpart of the X-ray source. Although we positioned the slit to cross this second object as well, the relative difference in brightness between both objects and the particularly poor value ( $2.3 \mathrm{arcsec})$ of the seeing for this exposure prevent a reliable extraction of the spectra. If both objects were physically associated, the angular separation would correspond to $670 \mathrm{AU}$.

\section{2. $N G C 2986-X 1$}

This field was not covered by SDSS; DSS images show a bright point-like object located near the position of the X-ray source. 
In March 2012, we took images of this field in $g, r$, and $i$ filters with the IAC 80 . The separation between the X-ray source and its optical counterpart is 0.26 arcsec. The magnitudes of the object are 17.96, 16.58, and $15.63 \mathrm{mag}$ in $g, r$, and $i$. The colours are compatible with those expected for an early M-class star. The spectra also shows the main features of this spectral class, so we classify the object as an M2 star and infer a distance of $\sim 283 \mathrm{pc}$. That distance gives an X-ray luminosity of $\sim 1.6 \times 10^{30} \mathrm{erg} \mathrm{s}^{-1}$. The slit was positioned to cross a brighter object as well (see Fig. 2), situated 10.3 arcsec to the north. The colours and spectrum show that this object is a $\mathrm{K}$ star. However, the uncertainties in the distances derived from the spectral types of both stars prevented us from determining if they form a physical pair.

\subsection{NGC 5322-X1}

The candidate optical counterpart is an isolated point-like object with a magnitude in SDSS of $i=18.9$. The spectrum shows several unambiguous absorption (CaII $\mathrm{H} \& \mathrm{~K}$, and $\mathrm{MgI}$ ) and emission (MgII, $\mathrm{H} \beta, \mathrm{H} \alpha$, OII, and $\mathrm{OIII})$ lines, from which we estimate a redshift of $0.4107 \pm 0.0002$; that confirms the photometric redshift of 0.4 estimated by Georgakakis et al. (2006). The spectral features allow a clear identification of the object as an active galactic nucleus (AGN). The redshift corresponds to a luminosity distance of $2229 \mathrm{Mpc}$, therefore the object has an absolute magnitude of $M_{i}=-22.8$ and an X-ray luminosity of $1.2 \times 10^{44} \mathrm{erg} \mathrm{s}^{-1}$.

\subsection{NGC 2500-X2}

The ULX catalogued by Swartz et al. is the source CXOJ080157.8+504339 listed by Evans et al. (2010). Several HII sources associated with NGC 2500 were identified by Hodge $\&$ Kennicutt (1983) at only a few arcsec distance from the X-ray source. The most likely optical counterpart is an isolated pointlike object with $i=18.47$ mag in SDSS images. There are several images in the HST archive confirming the optical identification. The spectrum shows as the most obvious features the Balmer lines $(\mathrm{H} \beta, \mathrm{H} \gamma$, and $\mathrm{H} \delta)$ and other broad emission lines such as MgII, and the forbidden narrow lines of OII and OIII. We estimate an $F W H M \sim 4000 \mathrm{~km} \mathrm{~s}^{-1}$ for the broad lines. There is no clear absorption features. From these spectral features we identify the object as an AGN at $z=0.5132$; this implies that the magnitude of the object is $M_{i}=-23.85$. From the redshift and the flux in X-ray we estimate a luminosity $9.1 \times 10^{43} \mathrm{erg} \mathrm{s}^{-1}$.

\subsection{NGC $4150-X 2$}

Swartz et al. catalogued two ULXs around the galaxy NGC 4150 in the positions $\mathrm{RA}=12^{\mathrm{h}} 10^{\mathrm{m}} 33.76^{\mathrm{s}}$, Dec $=+30^{\circ} 23^{\prime} 58.0^{\prime \prime}$ and $\mathrm{RA}=12^{\mathrm{h}} 10^{\mathrm{m}} 34.77^{\mathrm{s}}, \mathrm{Dec}=+30^{\circ} 23^{\prime} 58.3^{\prime \prime}$. Only this last one has a clear optical counterpart in SDSS images. This source was also catalogued as CXO J121034.7+302358 by Evans et al. (2010). There is a blue point-like isolated object with $i=18.74$ mag very close to the X-ray position. There is also an infrared counterpart, J12103468+3023578, in 2MASS. We tentatively identify that source as the optical counterpart of the ULXs. The spectrum shows strong broad MgII and Balmer lines $\left(\sim 3000 \mathrm{~km} \mathrm{~s}^{-1}\right)$ and narrow OII and OIII doublet in emission. These features are typical of AGN. We estimate a redshift of 0.5140 and from that an $\mathrm{X}$-ray luminosity of $5.6 \times 10^{44} \mathrm{erg} \mathrm{s}^{-1}$. The absolute magnitude of the object is $M_{i}=-23.59$.
The available HST images show other sources $(\geq 5$ mag fainter than the brighter source in the filter F814) through the N-NE at distances $\sim 1.5$ arcsec from the X-ray position. Although it is less likely due to their larger separation, they cannot be completely discarded as the counterpart of the ULX. Indeed, the brightest point-like source to the $\mathrm{N}$ would have $M_{i} \sim-6$ if it really were associated with NGC 4150, which is compatible with magnitudes found in other optical identifications (e.g. Tao et al. 2011).

\section{Discussion and conclusions}

We clarified the nature of five X-ray sources previously catalogued as ULXs in Chandra and XMM-Newton-based catalogues. For this we identified their optical counterparts in existing optical surveys and then characterized them spectroscopically. A missidentification in any of the cases is very unlikely. We have made a rough but conservative estimation of the probability of a chance alignment within a circle of radius 1 arcsec for a star (the optical counterpart of NGC 1068-X2, and NGC 2986$\mathrm{X} 1$ ), and for an AGN (the cases of NGC 5322-X1, NGC 2500$\mathrm{X} 2$ and NGC 4150-X2). The two optical counterparts identified as stars have magnitudes $r=12.80$ and 16.58. In SDSS DR9 the density of stars with magnitude $r \leq 16.58$ at galactic latitudes $>20$ degrees is $\sim 430$ per square degree, from which we obtained a probability for at least a chance alignment $\sim 2 \times 10^{-4}$. Abraham et al. (2012) used the SDSS photometric survey to estimate a density of QSOs at limiting magnitude $g \sim 20$ of 18 per square degree. Consequently, the probability to have at least one chance alignment in the three cases considered is $1.3 \times 10^{-5}$.

The main results can be summarized as follows:

1. In the five cases considered it is possible to assign a unique and unambiguous optical counterpart.

2. We showed that none of the five sources is a real ULX, and the association to their host galaxy was based on a pure random projection.

3. Two of the sources (NGC 1068-X2 and NGC 2986-X1) turn out to be foreground stars in our own galaxy with X-ray luminosities of $2.4 \times 10^{30}$ and $1.6 \times 10^{30} \mathrm{erg} \mathrm{s}^{-1}$.

4. The remaining three candidates (NGC 5322-X1, NGC 2500$\mathrm{X} 2$, and NGC 4150-X2) are AGNs at redshifts 0.4107, 0.5132 , and 0.5140 respectively.

5. Although the sample analysed here is clearly biased towards those ULX candidates with a bright point-like optical counterpart that reside in uncrowded regions, our results clearly pose the question of the relevance of studies in other spectral domains to achieve a correct census and statistics to unveil the ultimate nature of ULXs.

A programme to identify optical counterparts of several other ULXs is on-going at the $10.4 \mathrm{~m}$ GTC telescope (ORM, La Palma), and we expect to report the results in the near future.

Acknowledgements. The spectra presented in this paper were obtained with the Willam Herschel Telescope operated on the island of La Palma by the Isaac Newton group and the IAC in the Spanish Observatorio del Roque de los Muchachos. The images were made in service time with the IAC 80 telescope operated by the IAC on the island of Tenerife in the Spanish Observatorio del Teide. We thank the group of support astronomers and N. Lodieu. We thank J. Casares for the comments and discussion on the nature of some of the objects analyzed here. The paper has used the Hubble Legacy Archive, which is a collaboration between the Space Telescope Science Institute (STScI/NASA), the Space Telescope European Coordinating Facility (ST-ECF/ESA) and the Canadian Astronomy Data Centre (CADC/NRC/CSA). The author thanks an anonymous referee for several useful comments and suggestions that improved this work. 
C. M. Gutiérrez: Five fake ultra-luminous X-ray sources

\section{References}

Abraham, S., Philip, N. S., Kembhavi, A., Wadadekar, Y. G., \& Sinha, R. 2012, MNRAS, 419, 80

Arp, H., Gutiérrez, C. M., \& López-Corredoira, M. 2004, A\&A, 418, 877 Copperwheat, C., Cropper, M, Soria, R., \& Wu, K. 2005, MNRAS, 362, 79

Cseh, D., Corbel, S., Kaaret, P., et al. 2012, ApJ, 749, 17

Evans, I. N. 2010, ApJS, 189, 37

Farrell, S. A., Servillat, M., Pforr, J., et al. 2012, ApJ, 747, L13

Georgakakis, A. E., Georgantopoulos, I., \& Akylas, A. 2006, MNRAS, 366, 17

Ghosh, K. K., Saripalli, L., Gandhi, P., et al. 2009, AJ, 137, 3263

Grisé, F., Kaaret, P., Corbel, S., Feng, H., \& Cseh, D. 2012, ApJ, 745, 123

Gutiérrez, C. M. 2006, ApJ, 640, L17

Gutiérrez, C. M., \& López-Corredoira, M. 2005, ApJ, 622, L89
Gutiérrez, C. M., \& López-Corredoira, M. 2007, A\&A, 472, 87

Hodge, P. W., \& Kennicutt, R. C. 1983, AJ, 88, 296

King, A. R., Davies, M. B., Ward, M. J., Fabbiano, G., \& Elvis, M. 2001, ApJ, 552, L109

Kording, E., Falcke, H., \& Markoff, S. 2002, A\&A, 382, L13

Liu, J. 2011, ApJ, 192, 10

Liu, J. F., \& Bregman, J. N. 2005, ApJSS, 157, 59

López-Corredoira, M., \& Gutiérrez, C. M. 2006, A\&A, 454, 77

Stocke, J. T., Morris, S. L., Gioia, I. M., et al. 1991, ApJSS, 76, 813

Swartz, D. A., Soria, R., Tennant, A. F., \& Yukita, M. 2011, ApJ, 741, 49

Tao, L., Feng, H., Grise, F., \& Kaaret, P. 2011, ApJ, 737, 81

Tao, L., Kaaret, P., Feng, H., \& Grisé, F. 2012, ApJ, 750, 110

Yang, L., Feng, H., \& Kaaret, P. 2011, ApJ, 733, 118 